\title{
IDETC2010-28418
}

\section{DESIGN OF A ROBOTIC HAND WITH A BIOLOGICALLY-INSPIRED PARALLEL ACTUATION SYSTEM FOR PROSTHETIC APPLICATIONS}

\author{
Anthony L. Crawford \\ Idaho State University: College of Engineering \\ 921 South $8^{\text {th }}$ Ave. \\ Pocatello, ID 83209 \\ Phone: (208) 850-8233 \\ Email: crawanth@isu.edu
}

\author{
Dr. Alba Perez-Gracia \\ Idaho State University: College of Engineering \\ 921 South $8^{\text {th }}$ Ave. \\ Pocatello, ID 83209 \\ And: Institut de Robotica i Informatica Industrial (UPC/CSIC), \\ Barcelona, Spain \\ Phone: (208) 282-5655 \\ Email: perealba@isu.edu
}

\begin{abstract}
This paper presents the design of a robotic hand for prosthetic applications. The main characteristic of this robotic hand is its biologically-inspired parallel actuation system, which is based on the behavior/strength space of the Flexor Digitorum Profundus (FDP) and the Flexor Digitorum Superficialis (FDS) muscles. The design separates the strength space of the FDS and FDP muscles into a lighter strength region where finer manipulation and general approach tasks are executed, and a higher strength region where the more robust grasps are achieved. Two parallel actuator types and kinematic structures are designed to complement the requirements of both strength space regions.

This unique structure is intended to be driven by electromyographical (EMG) signals captured at the surface of the skin. The direct relation between signal and actuation system lends itself well to interpreting the EMG signals from the FDP and FDS muscles into effective task execution, with the goal of helping the user to achieve a good approximation of the full capabilities associated with the human hand, without compromising strength, dexterity, appearance, or weight; which are common issues associated with prosthetic hands.
\end{abstract}

\section{INTRODUCTION}

There have been many different approaches taken in the development of an effective prosthetic hand. These varying strategies often find themselves focusing on one of the following categories: implementing a new actuator type [1116], developing a more effective kinematic structure [18,23,34], integrating effective compliance [18,23-25], generating effective control strategies [25-31], and interpreting/conditioning input signals [25]. Advances in these areas have resulted in robotic hands that perform many tasks with a high similarity to that of the human hand, such as the DLR hand [33], I-Limb hand [11], Shadow hand [12], and Fluidhand [32] to name a few. However, a prosthetic hand that is nimble, quick, strong, lightweight, quiet, and efficient [1] has yet to be fully achieved.

The primary reason for the current state of prosthetic hands has been the complexity associated with the human hand as a result of its multiple bones and joints (Fig 1). This is further compounded by the fact that the human hand as a functioning unit does not just embody the palm and its digits but also the wrist, forearm muscles, nervous system, and the body's energy generation system. As a result, the entire prosthetic hand actuation structure (inputs, power, strength, kinematics, etc.) must fit in a significantly reduced volume compared to the human hand that it is replacing.

To address some of the challenges described above, this research implements a unique perspective of the FDS and FDP muscles' strength space in the human forearm and proposes a novel design and parallel actuation structure that complements this perspective. The goal is to create a direct relation between the forearm's EMG signals and the actuation system, in order to help the user achieve a good approximation of the full capabilities associated with the human hand in a compact design. 


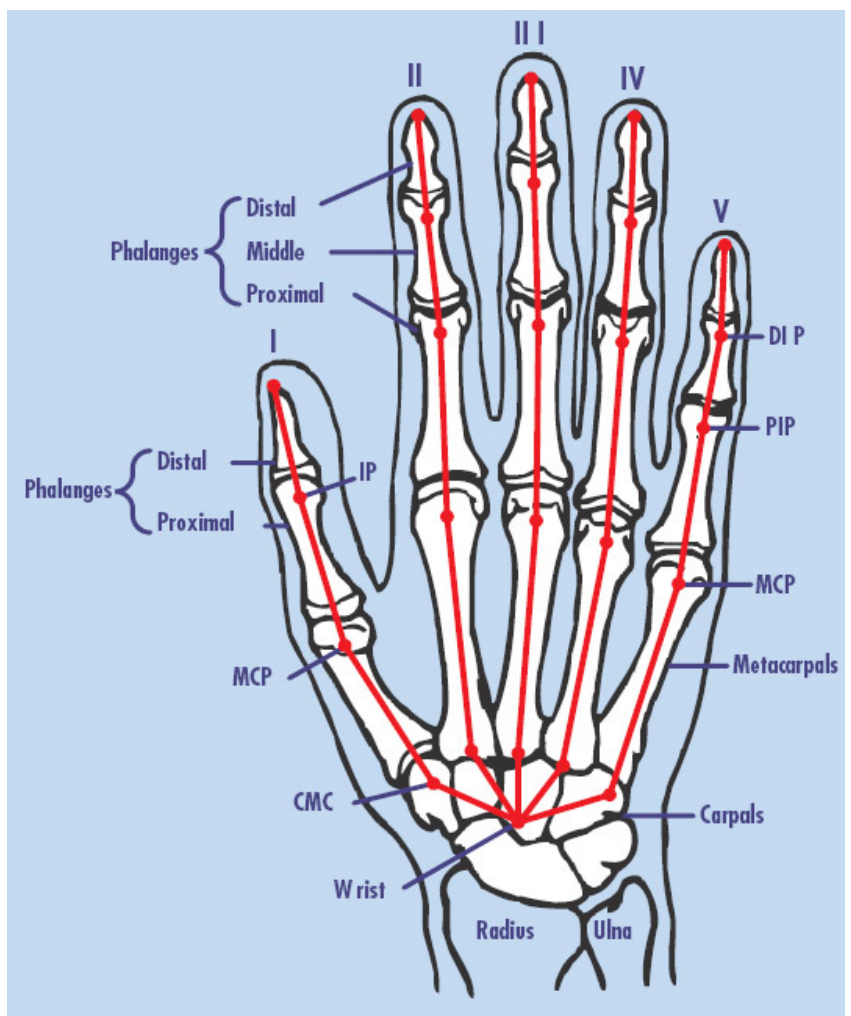

Fig 1: Joint/Bone composition of human hand [2]

Sections 2 and 3 of this paper describe the FDS and FDP muscles' strength space, how it relates to the human hand's capabilities, as well as the actuators and actuation structures of current prosthetic/robotic hands. Sections 4 and 5 will provide a description of the mechanical design and testing results that justify the design's ability to execute the strength space perspective developed in this paper. The paper will finally present conclusions associated with the testing results and an identification of future work.

\section{FDP MUSCLE'S KINEMATIC STRUCTURE AND STRENGTH SPACE}

The FDS and FDP muscles are the primary flexor muscles in the human finger and are primarily opposed by the extensor digitorum (ED) muscle. As shown in Fig 2. The FDP muscle is attached to the distal phalanx and is capable of full hand closure; it is considered to be the more active of both finger flexion muscles. The FDS muscle is attached to the middle phalanx and its full capacity is primarily achieved when activation of the DIP joint is not required or when full hand closure tasks require additional strength [3].

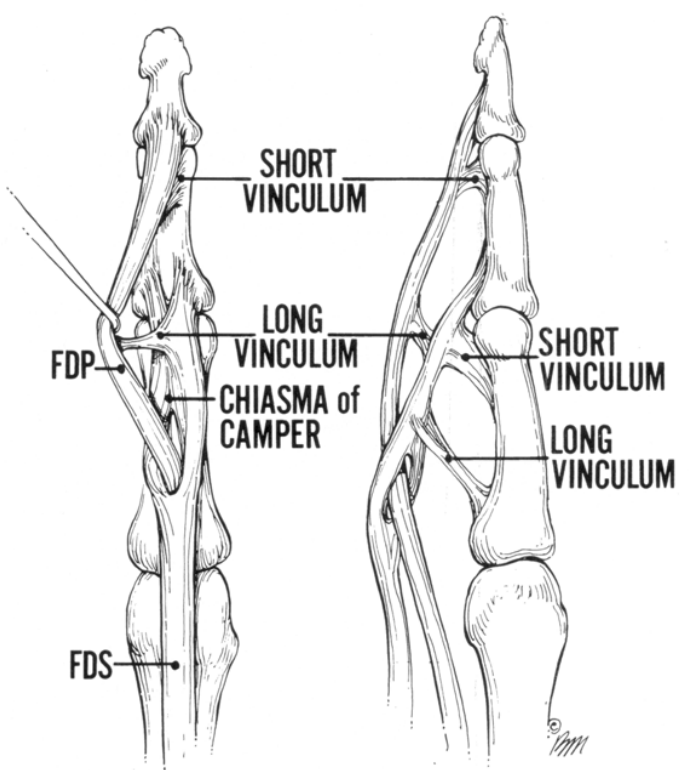

Fig 2: Graphic of FDS and FDP muscle in finger [4]

The strength space of the FDS and FDP muscles is shown in Fig 3. The figure demonstrates the normalized maximal force exertion of the FDS and FDP muscles (y-axes) during maximum force execution of the hand (x-axes). The FDP muscle is shown to reach its maximal force execution $(120 \mathrm{~N}$ [5]) at approximately $35 \%$ of the total flexural effort; however, the FDS muscle continues to exert force until it reaches its maximal force execution (240 N [5]) at about $100 \%$ of the total flexural effort. The FDS and FDP behavior can be attributed to the learned neurological activation of these muscles [6] as well as the finger's associated kinematic structure.

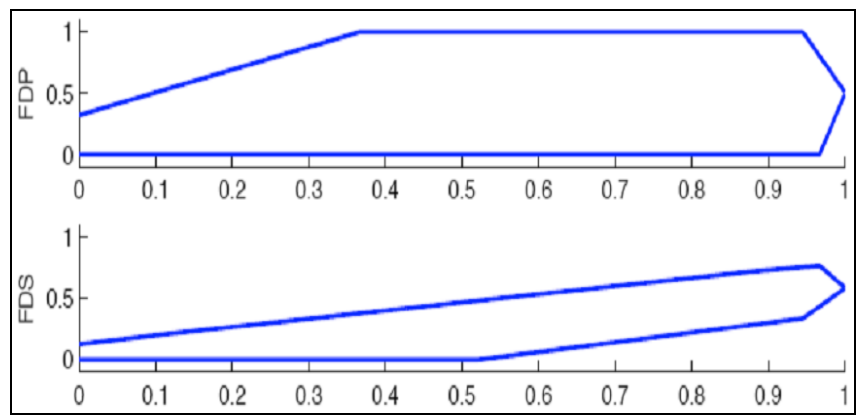

Fig 3: Strength space of FDS and FDP muscles [7]

The FDS and FDP strength space comprises the strength requirements for all the tasks that a hand must execute [8][9]. In general, most everyday tasks don't require extensive force but do require a certain amount of dexterity. For manipulation or approaching an object, the finger can employ both the FDS and FDP muscles to nimbly accommodate various shapes and execute both general and complex movement paths. Fig 3 shows that the FDS and FDP muscles are both active for activities below $35 \%$ of the maximum force capacity of the 
hand and are most likely employed during manipulation/object approaching movements.

Gripping tasks generally require less dexterity and more strength than manipulation and general object approach. The size and weight of the object as well as the characteristics of the grasp (e.g. friction between pads or force closure) determines how much force is required from the finger's strength space shown in Fig 3. It is to be noted that the actual act of the grip also generally requires much less complex finger motion than that of manipulation. Based on these observations, we divided the FDS and FDP strength space into the regions shown in Fig 4. In Fig 4 region 1 is populated by the more frequent dexterous tasks and region 2 is populated by the less frequent and more strength-based tasks.

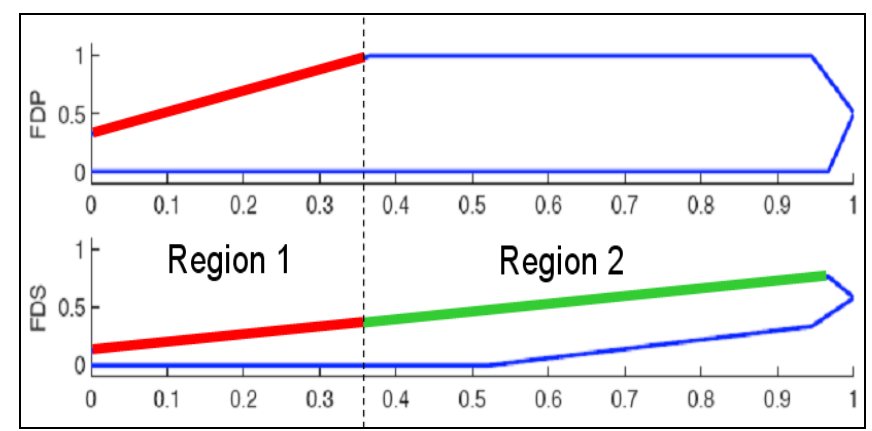

Fig 4: Divided Strength Space of FDS and FDP muscles

Though the bones, joints, and muscles of the thumb are somewhat different than that of the finger, the same FDS and FDP strength space division philosophy is applied in the mechanical design of the prosthetic thumb.

\section{ACTUATION STRUCTURES}

To the authors' knowledge, all currently developed prosthetic/robotic hands use a single actuator type to execute all the tasks embodied in the FDS and FDP strength space. This technique results in the shortcomings of the chosen actuator being carried throughout the strength space, let it be pneumatic, electromechanical, ultrasonic, or shape memory alloy. This could include excessive size and weight, or reduced time response and energy inefficiency to name a few.

The actuator types used in current robotic/prosthetic hands and considered in this design included electromagnetic [10][11], pneumatic [12][13], hydraulic [14], ultrasonic [15], and shape memory alloy [16]. Although all the listed actuator types have been employed in multiple prosthetic hand designs based on their advantageous features, one or more shortcoming (weight, noise, size, efficiency, and speed) have been accepted as well.

Prosthetic hands have employed the above actuator types into two general types of kinematic structures. These two structures are referred to as fully actuated and underactuated. The underactuated structure often uses a single input to actuate the multiple joints and essentially wraps the phalanges of the finger around an object [17]. Typically this is achieved by a flexible tendon routed through the finger structure which allows one of the finger's phalanges to be stopped by the object without preventing the remaining phalanges from continuing to wrap around the target

Some underactuated structures couple all three degrees of freedom associated with finger flexure to one actuator. However, there are structures where the underactuation exists only between the PIP and DIP joints. This behavior is typically executed by the human hand and will be referred to herein as nearly fully actuated. One example of this type of nearly fully actuated structure is that adopted by Yamano, Takemura and Maeno [15]. Dollar and Howe present many other types of couplings that have been employed in various underactuated hands [18]. The primary disadvantage of these structures is the lack of manipulation capabilities.

Fully actuated or nearly fully actuated structures do allow the greater manipulability lacking in underactuated structures. The consequence of this flexibility is the increased number of motors required to actuate these degrees of freedom, where each motor must also be of adequate size to apply the required forces. This in turn increases the size, weight, and control complexity of the prosthetic hand.

Few prosthetic hands are actually fully actuated. This could arise from the complexity that is introduced in the design or from the fact that the tasks which are to be performed are modeled after the underactuated human hand for grasping actions only. One hand that does fully actuate the finger's degrees of freedom is the UB-3 hand [19]. In this finger each phalange has a tendon attached to it and is able to actuate all flexing degrees of freedom independently.

In the design presented in this paper, the actuators and actuation structure were chosen to specifically complement the task characteristics of each region shown in Fig 4. The smaller, faster, and efficient electromagnetic motors are incorporated into a nearly fully actuated kinematic structure and chosen to perform the tasks associated with region 1 . The quiet, lightweight, strong shape memory actuators implemented in a parallel underactuated structure were selected to provide sufficient strength to the system when required. The complementary function of both actuation systems provides the prosthetic hand with a broad capability for grasping and manipulating actions while trying to optimize actuator size and performance.

\section{PROSTHETIC HAND DESIGN}

The developed design is shown in Fig 5. The design is dimensionally consistent with that of an average male human hand [2] and possesses the same degrees of freedom. The anthropomorphic aspect of the hand is intended to enhance the amputee's acceptance and usability. The DIP and PIP joints of the finger and the IP and MCP joints of the thumb are coupled. This is achieved by connecting a single actuator to both the PIP joint (bevel gears) and DIP joint (pulley connection on metacarpal phalange). This coupling technique is common among many prosthetic/robotic hands as noted in the section above. 


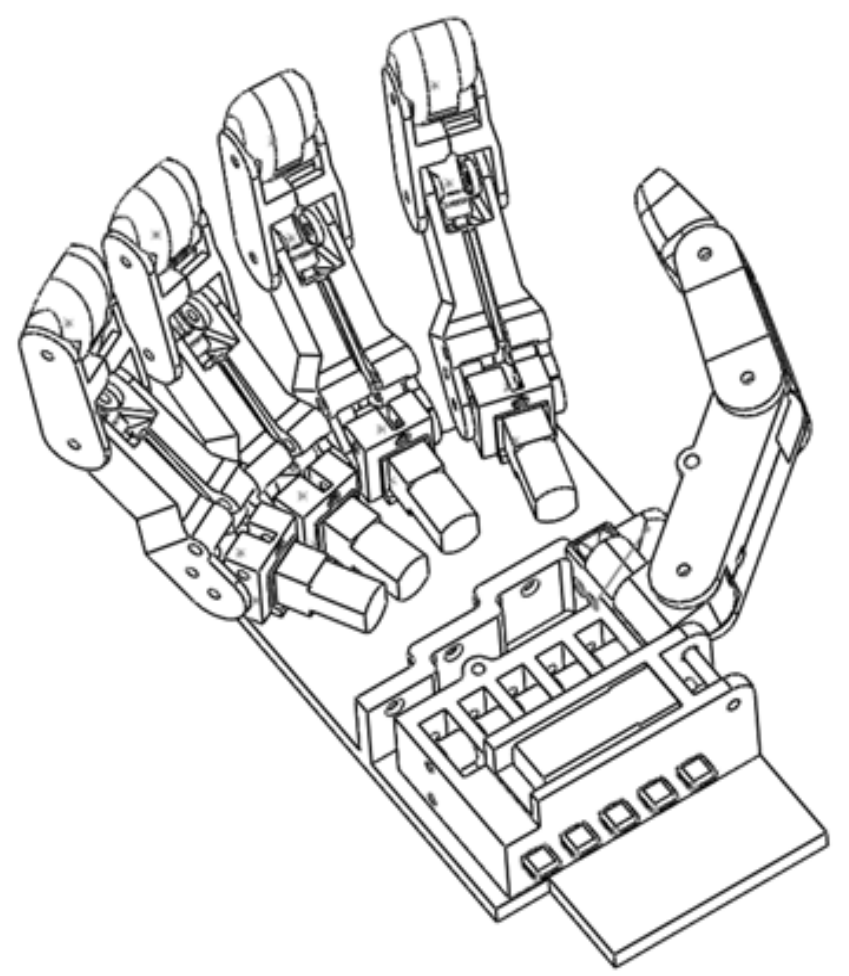

Fig 5: Prosthetic hand design

The developed parallel actuation structures discussed in the previous section are shown in Fig 6 and Fig 7 for the finger and Fig 8 and Fig 9 for the thumb. The movements associated with region 1 in Fig 4 are achieved by two DC motors. The DC motors actuating the coupled DIP/PIP joints of the finger and IP/MCP joints of the thumb are embedded in the proximal phalange of the finger and the metacarpal phalange of the thumb. The DC motor in the metacarpal phalange of the finger actuates the horizontal degree of freedom of the MCP joint. The DC motor at the base of the thumb actuates the CMC joint to obtain an approximation of the abduction/adduction motion. The second degree of freedom of the finger's MCP joint (abduction/adduction) is only subject to compliance without actuation. The second degree of freedom in the thumb's CMC joint (flexion/extension) is actuated by the region 2 actuation structure.

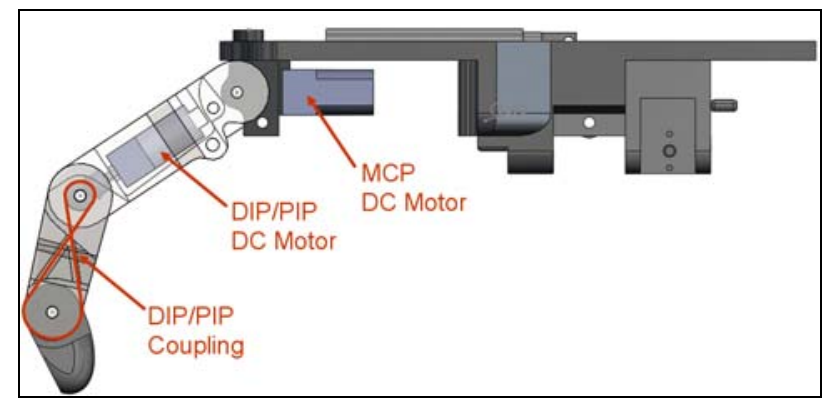

Fig 6: Region 1 actuation scheme for the finger

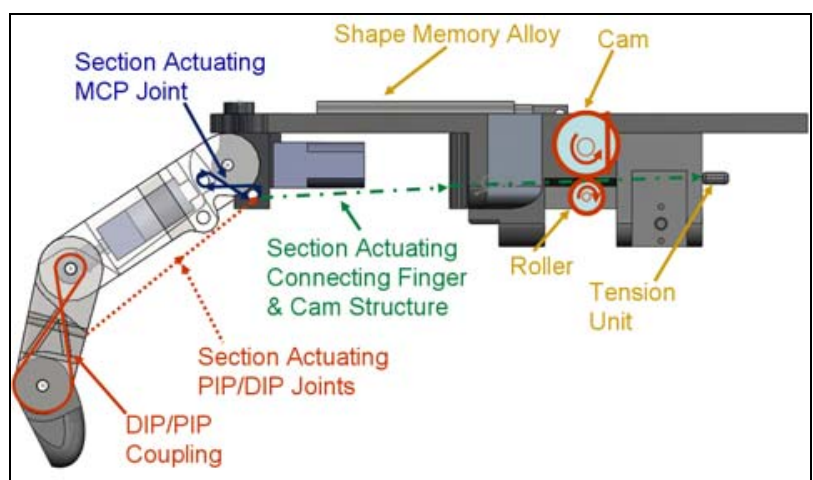

Fig 7: Region 2 actuation scheme for the finger

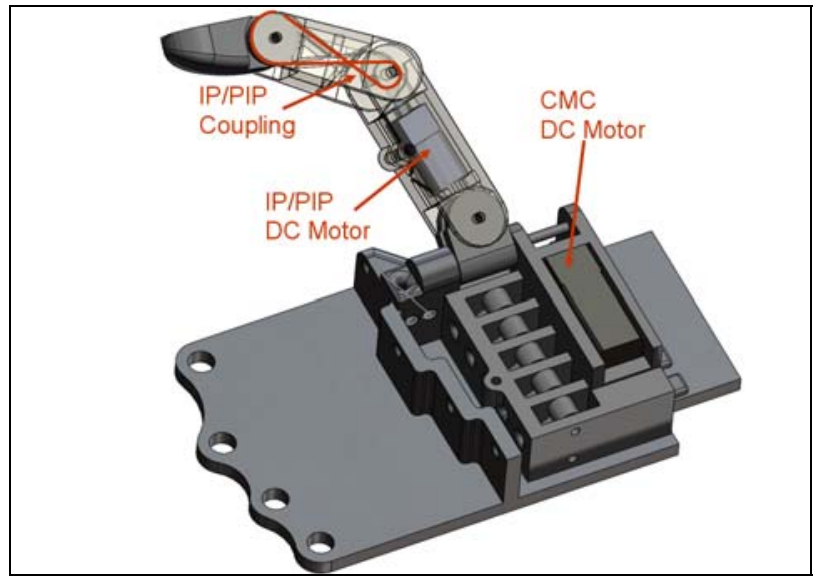

Fig 8: Region 1 actuation scheme for the thumb

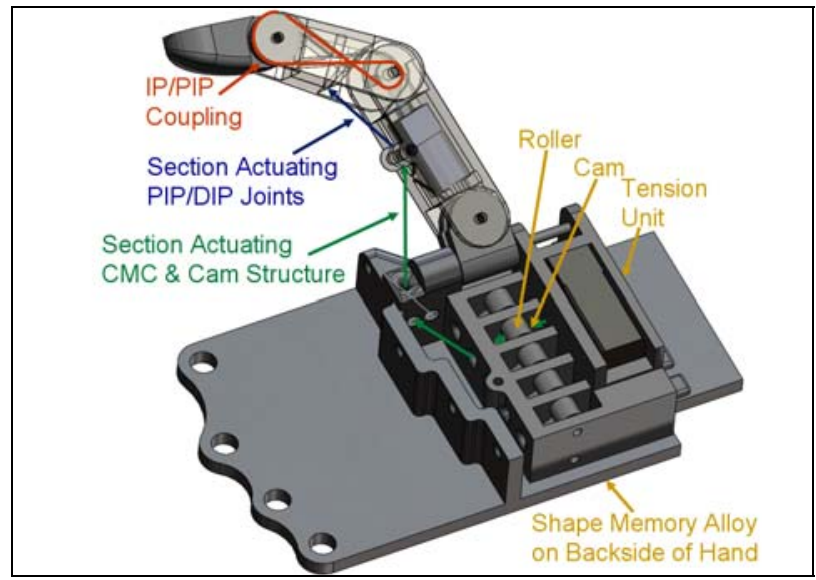

Fig 9: Region 2 actuation scheme for the thumb

The actuation structure corresponding to region 2 in Fig 4 for the finger includes a light cable that passes over two restraining shafts in the MCP joint of the finger, coils in the proximal phalange, and embeds in the middle phalange. The string is kept in light tension by a tension unit at the back of the hand while the region 1 actuation structure is active. When region 2 actuation is required the shape memory alloy actuates a spring loaded cam which in turn pinches the string between itself and a roller beneath it. As the shape memory alloy continues to actuate, the cam introduces the additional force required for 
region 2 tasks. At task completion the electric signal causing the shape memory alloy to heat up is stopped and the DC motors and cam spring extend the shape memory alloy back to its original state. The spring loaded cam mechanism is more definitively shown in Fig 10 and Fig 11.

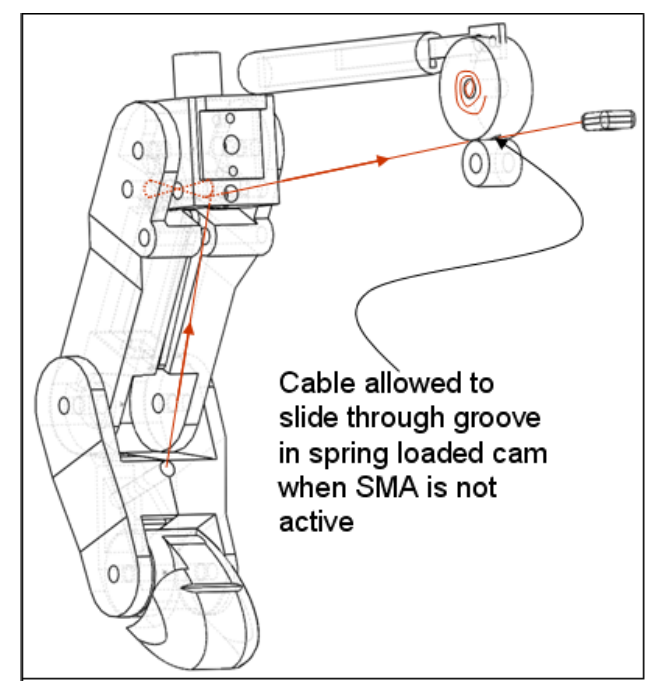

Fig 10: Region 2 spring loaded cam mechanism prior to SMA actuation (thinner line representing cable corresponds to lower tension applied by tensioner)

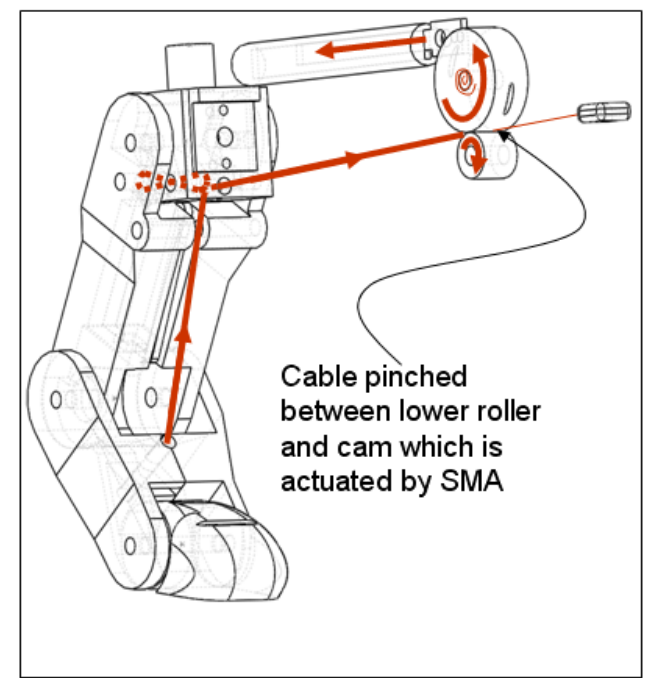

Fig 11: Region 2 spring loaded cam mechanism during SMA actuation (thicker line representing cable corresponds to additional tension applied by SMA via cam mechanism)

The thumb's region 2 actuation structure is similar to that of the finger's region 2 actuation structure. However, unlike the finger, this structure actuates the degree of freedom at the CMC joint that is not actuated by the DC motor. This is based on the observation that this degree of freedom is more apposing of the fingers during tasks that would require additional force (power grasp, high force pinch grasp, lateral grasp, etc. [8]).
The design shown in this section has been manufactured using a rapid prototyping machine. The prototype, equipped with the actuators and transmission system, can be seen in the Results and Discussion section.

\section{RESULTS AND DISCUSSION}

The experiment associated with this research was performed to validate the designed actuation structure's ability to span a two-region strength space similar to the one identified above for the human hand. The actuators used in the prototype were Pololu 298:1 Micro Metal Gearmotor HP DC motors [21] and the Electric Piston SMA actuator from Raychem [22]. These actuators are relatively inexpensive and the implementation of more expensive actuators could further enhance the values reported below.

The experiment consisted of having the finger and the thumb grip a FSR sensor fastened to a dense foam ball in the large grip and then the close grip configurations as shown in Fig 12 through Fig 15. The large grip setup simulated the finger/thumb performing a more robust grasp on a larger object and the small grip setup simulated the actuation structure of the finger/thumb grasping smaller objects or performing the more dexterous pinch or lateral grasp. The values obtained experimentally are compared to the expected calculated values using a simple static calculation of the reaction force required at the FSR sensor to resist the stall torque of the DC motors and the measured tension provided by the SMA.

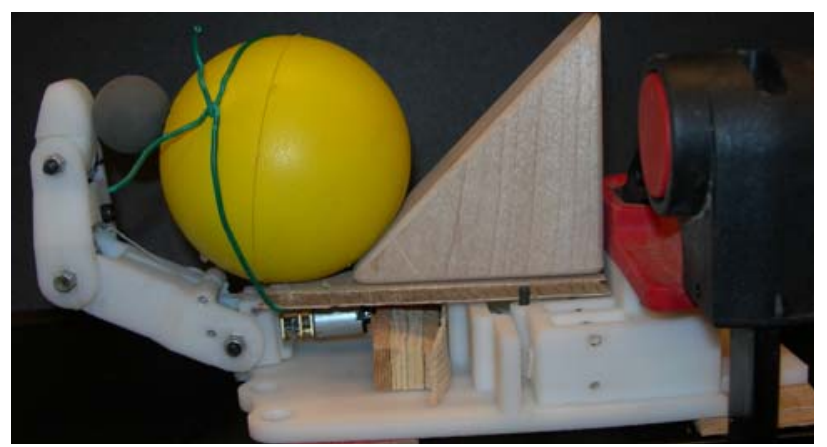

Fig 12: Finger in large grip configuration.

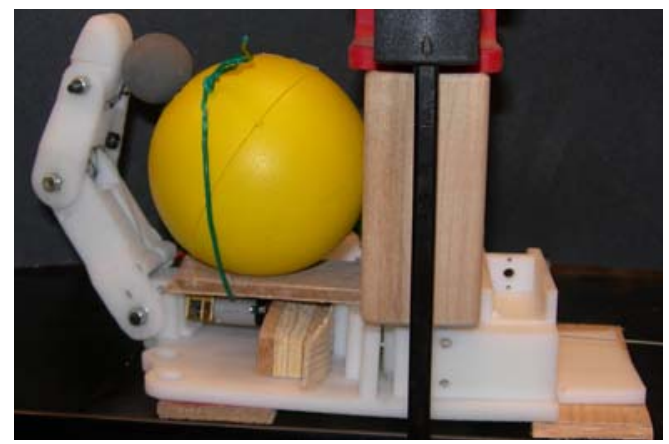

Fig 13: Finger in close grip configuration. 


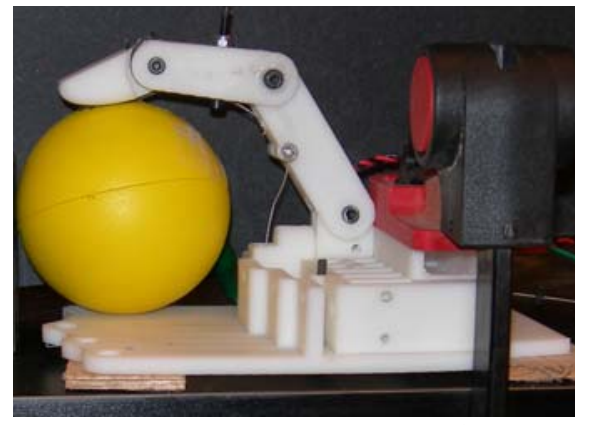

Fig 14: Thumb in close grip configuration.

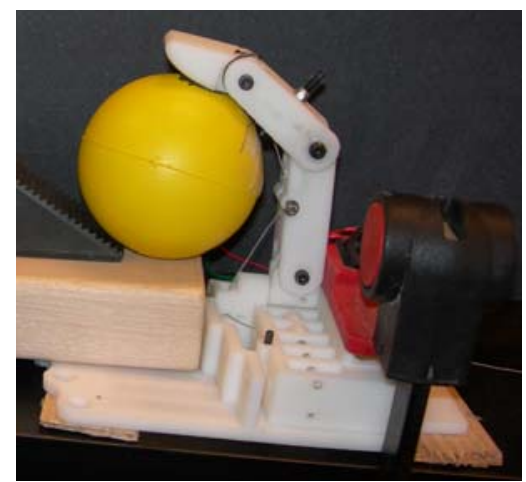

Fig 15: Thumb in large grip configuration.

The experimental values were compared to the expected ones using static calculations based on the free body and geometric diagrams shown in Fig 16 through Fig 19 (only large grip configurations are shown). The static equations used are shown in (1) and (2) for the finger and thumb and operate by solving for the forces/torques of each joint from the distal phalange to the knuckle in terms of the variable RF value then using the equations to solve for RF. The tension applied by the SMA in the experiments was multiplied by the "T" vectors in Fig 16 and Fig 18. The stall torque applied by the DC motors (90 ozin) replaced the M1 and M2a/M2b variables. As described in the design section M2a and M2b are coupled and their relationship is shown in (3). The gravitational forces generated by each phalange was applied at the center of the link in the downward direction using the mass values for the finger of $\left(M_{\text {prox }}=21.6 \mathrm{~g}, M_{\text {mid }}=9.45 \mathrm{~g}, M_{\text {dist }}=6.7 \mathrm{~g}\right)$ and for the thumb of $\left(M_{\text {met }}=33.3 \mathrm{~g}, M_{\text {prox }}=10.5 \mathrm{~g}, M_{\text {dist }}=8.5 \mathrm{~g}\right)$.

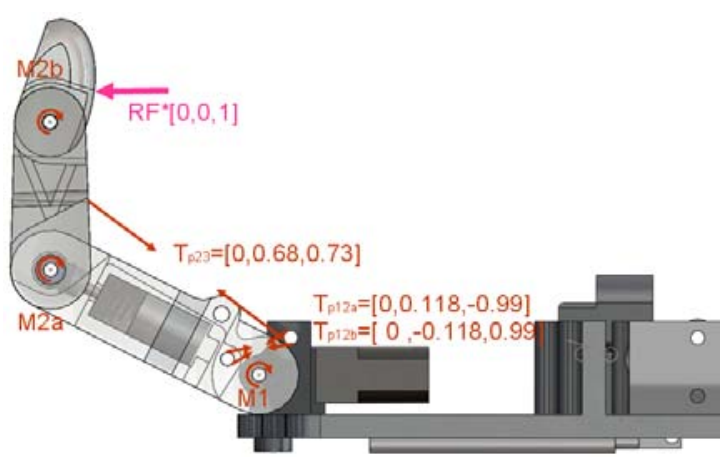

Fig 16: Free body diagram of large grip finger

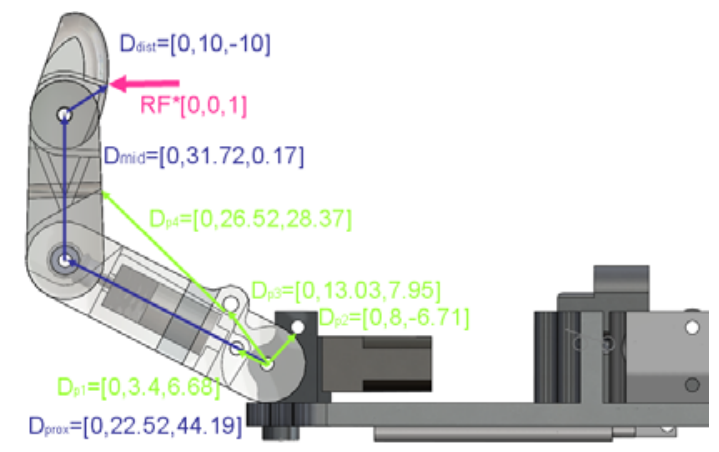

Fig 17: Geometric dimensions of large grip finger

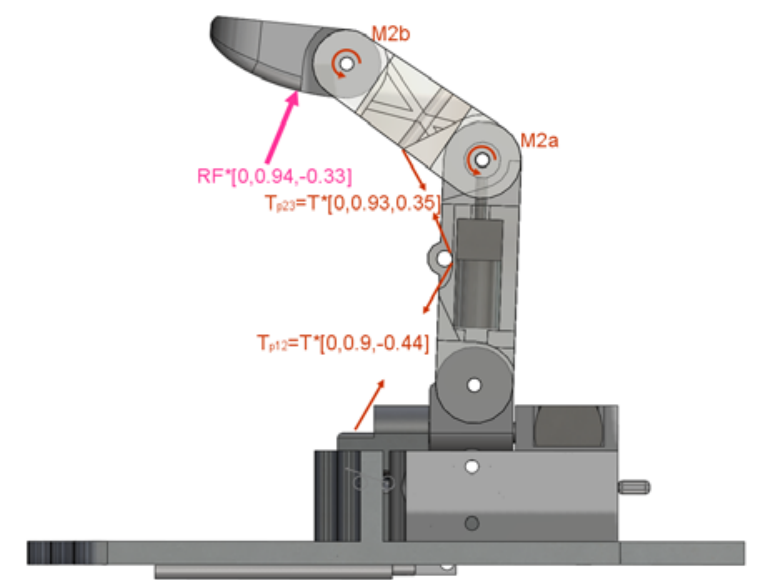

Fig 18: Free body diagram of large grip thumb

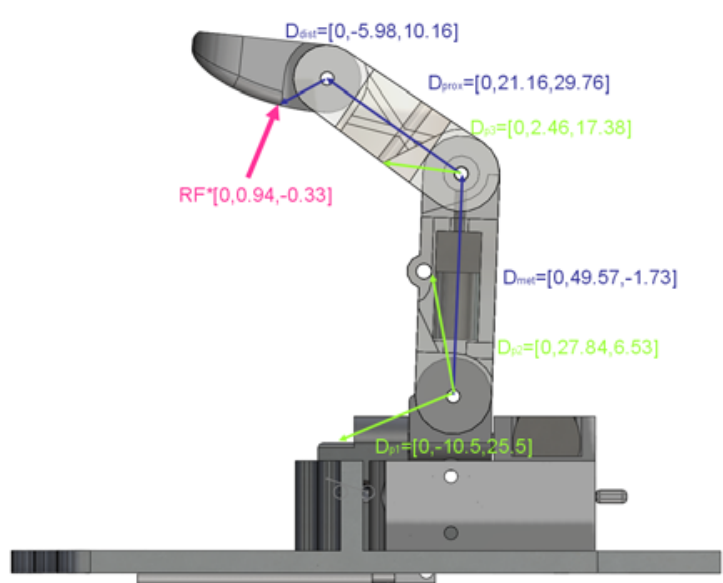

Fig 19: Geometric dimensions of large grip thumb

$$
\begin{aligned}
& \sum M=0 \\
& \sum F=0 \\
& M 2 a+\frac{8}{14} M 2 b=90 o z-i n
\end{aligned}
$$


Each of the four configuration setups were performed three times with each actuator type individually and then with them combined. The results are shown in Table 1 where the columns correspond to the resulting forces from the SMA, DC motors, the results of the SMA and DC motors column summed together, the experienced force when the two actuator types are physically applied at the same time, and the value calculated using basic statics. The table shows that when applied individually, the resulting forces from the SMA are for the most part larger than the applied DC motor forces for all four configurations. The average combined forces for the finger are generally greater than the experienced but the values for the thumb are approximately the same.

The finger behavior is expected because the SMA was only partly isolated from the motors as the motors were still employed during the SMA test in order to maintain the structure of the finger against the ball prior to testing. This resulted in the SMA having to press against the ball and overcome the minor resistance presented by the motors. The thumb behavior was expected as well due to the slightly compliant units isolating the motor during the motor test absorbing some of the applied motor force. The calculated forces where also expectedly higher than the measured data as friction from the system was not incorporated in their development.

It is to be noted that the combined (calculated) values for the thumb are only calculated for the SMA input and the DC Motor experimental value has been added to the calculated value. This was done because a part of the component in the prototype thumb broke causing the gearing to not mesh appropriately, giving significantly smaller force values (approximately $3 \mathrm{~N}$ ) than those calculated (approximately $14 \mathrm{~N}$ ).

Table 1: Finger \& Thumb Testing Results

Finger M1 \& M2 \& SMA (Newtons)

\begin{tabular}{|c|c|c|c|c|c|}
\hline Finger CG & SMA & DC Motors & $\begin{array}{c}\text { Combined } \\
(\text { Sum })\end{array}$ & $\begin{array}{c}\text { Combined } \\
\text { (Experimental) }\end{array}$ & $\begin{array}{c}\text { Combined } \\
\text { (Calculated })\end{array}$ \\
\hline Average & 7.75 & 9.78 & 17.52 & 15.23 & 19.11 \\
\hline Std Dev & 0.21 & 0.22 & 0.15 & 0.20 & 0.12 \\
\hline \multicolumn{7}{|l|}{} \\
\hline Finger LG & SMA & DC Motors & $\begin{array}{c}\text { Combined } \\
\text { (Sum) }\end{array}$ & $\begin{array}{c}\text { Combined } \\
\text { (Experimental) }\end{array}$ & $\begin{array}{c}\text { Combined } \\
\text { (Calculated) }\end{array}$ \\
\hline Average & 9.81 & 8.67 & 18.48 & 17.07 & 19.72 \\
\hline Std Dev & 0.16 & 1.14 & 1.27 & 1.53 & 0.55 \\
\hline
\end{tabular}

Thumb M1 \& SMA (Newtons)

\begin{tabular}{|c|c|c|c|c|c|}
\hline Thumb CG & SMA & DC Motor & $\begin{array}{c}\text { Combined } \\
\text { (Sum) }\end{array}$ & $\begin{array}{c}\text { Combined } \\
\text { (Experimental) }\end{array}$ & $\begin{array}{c}\text { Combined } \\
\text { (Calculated) }\end{array}$ \\
\hline Average & 4.70 & 1.24 & 5.94 & 5.60 & $5.72^{*}$ \\
\hline Std Dev & 0.90 & 0.35 & 0.86 & 1.14 & 0.31 \\
\hline \multicolumn{7}{|l|}{} \\
\hline Thumb LG & SMA & DC Motor & $\begin{array}{c}\text { Combined } \\
\text { (Sum) }\end{array}$ & $\begin{array}{c}\text { Combined } \\
\text { (Experimental) }\end{array}$ & $\begin{array}{c}\text { Combined } \\
\text { (Calculated) }\end{array}$ \\
\hline Average & 4.56 & 3.45 & 8.01 & 8.18 & $9.38^{*}$ \\
\hline Std Dev & 1.85 & 0.45 & 2.45 & 0.39 & 9.60 \\
\hline
\end{tabular}

* Values combined from calculated SMA values and measured DC Motor values

\section{CONCLUSIONS AND FUTURE WORK}

This paper presents a novel design and actuation system for a prosthetic hand. The actuation structure was shown to effectively span a two-region strength space to execute grip configurations similar to those found in the three primary grips of the human hand (power, pinch, and lateral). The design also showed the ability of an underactuated and nearly fully actuated kinematic structure to exist in a single actuation system using remarkably different types of actuators, without compromising the required size and weight of the prosthetic hand.

According to these results, the parallel actuation structure is a good starting point for the design. However, the complete testing of the parallel actuation system's performance requires the use of human-generated driving signals for different grasping and manipulation actions.

Future work includes further testing, the full identification of frictional losses in the system for static grasping and the use of the dynamical model of the hand for manipulation tasks, in order to improve the design. More precisely selected actuators will be used in the final design. The stronger actuators will add the appropriate scale to the developed strength space so as to mimic the force generation capabilities of the system as well as the strength space form proven in this paper. Other components of the project also being performed in parallel to the hand design include integrating the appropriate hardware to drive the device, interpreting the EMG signals in a manner appropriate to distinguish region 1 and region 2 performance requests by the FDS and FDP muscles, and further grasping studies.

\section{ACKNOWLEDGMENTS}

This research was performed under an award/contract from Telemedicine Advanced Technology Research Center (TATRC), of the U.S. Army Medical Research and Materiel Command (USAMRMC) of the U.S. Department of Defense.

\section{REFERENCES}

[1] Bicchi, A., 2000, "Hands for dexterous manipulation and robust grasping: a difficult road toward simplicity”, IEEE Transactions on Robotics and Automation, 16(6):652-662.

[2] Foegelle, M., Li, K., Pavacic, A., Moller, P., “Test \& Measurement: Developing a Standard Hand Phantom for Wireless Testing,” [Online]. Available:

http://www.wirelessdesignmag.com/ShowPR.aspx?PUBCODE=055\&AC $\underline{\mathrm{CT}}=0029956 \& \mathrm{ISSUE}=0811 \& \mathrm{RELTYPE}=\mathrm{tm} \& \mathrm{PRODCODE}=\mathrm{W} 0260 \& \mathrm{PR}$ ODLETT $=$ A\&CommonCount $=0$,

[3] Norkin, C., Levangie, P., 1992, Joint Structure \& Function: A Comprehensive Analysis: Second Edition, F. A. Davis Publications, 262299.

[4] Nelson, D. L. “Tendon Laceration Page," [Online]. Available: http://www.davidlnelson.md/Tendon_laceration.htm,

[5] Valero-Cuevas, F. J., Towles, J. D., Hentz, V. R., "Quantification of fingertip force reduction in the forefinger following simulated paralysis of extensor and intrinsic muscles,” Journal of Biomechanics 33 (2000) 16011609.

[6] Kevin G. Keenan, K. G., Veronica J. Santos, V. J., Venkadesan, M. and Valero-Cuevas, F.J., Maximal Voluntary Fingertip Force Production Is Not Limited by Movement Speed in Combined Motion and Force Tasks, The Journal of Neuroscience, July 8, 2009 • 29(27):8784-8789.

[7] Kutch, J. J., Valero-Cuevas, F. J., "All muscles are redundant but some are less redundant than others,” poster presentation for USC Viterbi School of Engineering Brain-Body Dynamics Lab. 
[8] Perez-Gracias, M. A., http://progeny.isu.edu/ alba/, Idaho State University.

[9] Cutkosky, M.R., 1989, “On grasp choice, grasp models, and the design of hands for manufacturing tasks", IEEE Transactions on Robotics and Automation, 5(3):269-279.

[10] Butterfass, J.; Fischer, M.; Grebenstein, M.; Haidacher, S. and Hirzinger, G.., 2004, "Design and Experience with DLR Hand II", Proceedings of the World Automation Congress 2004, Seville, Spain, 2004.

[11] Touch Bionics Inc [Online]. Available: www.touchbionics.com

[12] Shadow Robot Company [Online]. www.shadowrobot.com/hand/papers.shtml

[13] Fite, K., Withrow, T., Shen, Z., Wait, K., Mitchell, J., Goldfarb, M., 2008, "A Gas-Actuated Anthropomorphic Prosthesis for Transhumeral Amputees,” IEEE Transactions on Robotics, 24(1):159:169.

[14] Pylatiuk, C., Schulz, S., Kargov, A., Bretthauer, G., 2004, "Two Multiarticulated Hydraulic Hand Prostheses," Artifical Organs, 28(11),980:986.

[15] I. Yamono, T. Maeno, 2005, "Five-fingered Robot Hand using Ultrasonic Motors and Elastic Elements," Proceedings of the 2005 IEEE International Conference on Robotics and Automation, Barcelona, Spain, Aprill 2006.

[16] Andrianesis, K., Tzes, A., "Design of an Anthropomorphic Prosthetic Hand Driven by Shape Memory Alloy Actuators," Proceedings of the $2^{\text {nd }}$ Biennial IEEE/RAS-EMBS International Conference on Biomedical Robotics and Biomechatronics, Scottsdale, AZ, USA, October 19-22, 08.

[17] Carrozza, M., Cappielo, G., Micera, S., Edin, B., Beccai, L., Cipriani, C., 2006, "Design of a cybernetic hand for perception and action," Biol Cybern (2006) 95:629-644.

[18] Dollar, A.M., and Howe, R.D., 2006, "Joint coupling design of underactuated grippers”, Proc. of the ASME 2006 International Design Engineering Technical Conferences and Computers and Information in Engineering Conference, September 10-13, 2006, Philadelphia, PA, USA.

[19] Dollar Lotti, F., Tiezzi, P., Vassura, G., 2005, “Development of UB Hand 3: Early Results,” Proceedings of the 2005 IEEE International Conference on Robotics and Automation, Barcelona, Spain, April 2005.

[20] Hollerbach, J., "ME 6220: Introduction to Robotics", Chapters 9 \& 10 of Course Material, 2007.

[21] Pololu Robotics \& Electronics, "298:1 Micro Metal Gearmotor HP," [Online] Available: http://www.pololu.com/catalog/product/994

[22] Electric Piston Instruction Manual, [Online]. Available: www.robotstore.com/download/EPiston_Instr_V12.pdf

[23] Carrozza, M., Cappiello, G., Stellin, G., Zaccone, F., Vecchi, F., Micera, S., Dario, P., "A Cosmetic Prosthetic Hand with Tendon Driven UnderActuated Mechanism and Compliant Joints: Ongoing Research and Preliminary Results,” Proc. of the 2005 IEEE International Conference on Robotics and Automation, April 2005, Barcelona, Spain.

[24] Vanderniepen, I., Van Ham, R., Van Damme, M., Lefeber, D., "Design of a powered elbow orthosis for orthopaedic rehabilitation using compliant actuation,” Proc. of the $2^{\text {nd }}$ Biennial IEEE/RAS-EMBS International Conference on Bimedical Robotics and Biomechatronics, October 19-22, 2008, Scottsdale, AZ, USA.

[25] Zollo, L., Roccella, S., Guglielmelli, E., Carrozza, M., Dario, "Biomechatronic Design and Control of an Anthropomorphic Artificial Hand for Prosthetic and Robotic Applications," IEEE/ASME Transactions on Mechatronics, Vol. 12, No. 4, August 20007.

[26] Engeberg, E. D., Meek, S., "Improved Grasp Force Sensitivity for Prosthetic Hands Through Force-Derivative Feedback," IEEE Transactions on Biomedical Engineering, Vol. 55, No. 2, February 2008.

[27] Engeberg, E. D., Meek, S., "Model-Based Feedforward Derivative Compensation for Prosthetic Hands," Proc. of the $2^{\text {nd }}$ Biennial IEEE/RAS-EMBS International Conference on Bimedical Robotics and Biomechatronics, October 19-22, 2008, Scottsdale, AZ, USA.

[28] Engeberg, E. D., Meek, S. G., Minor, M. A., "Hybrid Force-Velocity Sliding Mode Control of a Prosthetic Hand,” IEEE Transactions on Biomedical Engineering, Vol. 55, No. 5, February 2008.

[29] Cipriani, C., Zaccone, F., Micera, S., Carrozza, M., "On the Shared Control of an EMG-Controlled Prosthetic Hand: Analysis of UserProsthesis Interaction,” IEEE Transactions on Robotics, Vol. 24, No. 1, February 2008.
[30] Blank, A., Okamura, A. M., Kuchenbecker, K. J., "Effects of Proprioceptive Motion Feedback on Sighted and Non-Sighted Control of a Virtual Hand Prosthesis,” Symposium on Haptic Interfaces for Virtual Environments and Teleoperator Systems, March 13-14, 2008, Reno, Nevada, USA.

[31] Robinson, D. W., Pratt, G. A., "Force Controllable Hydro-Elastic Actuator,” Proc. of the 2000 IEEE International Conference on Robotics and Automation, April 200, San Fransico, CA, USA.

[32] Pylatiuk, C., Schulz, S., Kargov, A., Bretthauer, G., 2004, "Two Multiarticulated Hydraulic Hand Prostheses,” Artifical Organs, 28(11),980:986.

[33] Institute of Robotics and Mechatronics [Online]: http://www.dlr.de/rm/en/desktopdefault.aspx/tabid-3975/6161 read-245/

[34] Winfrey, Rex C., 2008, "Prosthetic Hand Having a Conformable, Compliant Grip, and Opposable, Functional Thumb,” Patent 7,361,197, April 22, 2008. 